Desi gn of Thr ee- Di mensi onal Opt i cal Ci r cui t Devi ces by Usi ng Topol ogy Opt i mizat i on Met hod wi th Funct i on- Expansi on- Based Ref ract i ve I ndex Di st ri but i on

\begin{tabular}{|l|l|}
\hline 著者 & $\begin{array}{l}\text { YASUl Takashi, TSUJ Y Yasuhi de, SUG SAKA } \\
\text { Jun- i chi r o, H RAYAMA Koi chi }\end{array}$ \\
\hline $\begin{array}{l}\text { j our nal or } \\
\text { publ i cat } \mathrm{i} \text { on } \mathrm{t} \text { i t l e }\end{array}$ & Journal of Li ght wave Technol ogy \\
\hline vol une & 31 \\
\hline number & 23 \\
\hline page range & $3765-3770$ \\
\hline year & $2013-12-01$ \\
\hline URL & ht t p: //hdl . handl e. net /10258/00009486 \\
\hline
\end{tabular}




\title{
Design of Three-Dimensional Optical Circuit Devices by Using Topology Optimization Method with Function-Expansion-Based Refractive Index Distribution
}

\author{
Takashi Yasui, Member, IEEE, OSA, Yasuhide Tsuji, Member, IEEE, OSA, Jun-ichiro Sugisaka, \\ and Koichi Hirayama, Senior Member, IEEE
}

\begin{abstract}
We extend topology optimization method with function-expansion-based refractive index distribution to optimization for three-dimensional optical circuits, in which a refractive index distribution in a design region is expressed by an expansion with some analytical functions. Three-branch optical waveguides have been optimized as numerical examples. Equally branching three-branch waveguides are achieved using our method. A limitation of topology optimization in two dimensions and dependency of initial structure are also shown.
\end{abstract}

Index Terms-Finite-element method, three-dimensional analysis, optical waveguide, topology optimization.

\section{INTRODUCTION}

$\mathbf{I}$ $\mathrm{N}$ the recent progress of optical communication systems, many kinds of high-performance and compact optical waveguide devices have been developed. Especially, silicon nanopohotnic waveguides have great possibility to realize such devices, and extensive researches on these waveguides are being carried out.

Recently, topology optimization methods have begun to be used to realize high-performance microwave and photonic waveguide devices [1]-[12]. These methods can simultaneously deal not only with geometric form but also with topological configuration, so we can find out optimum structures that realize desired transmission characteristics.

In topology optimization, the density method was widely used to express refractive index distribution [1]-[3]. However, the topology optimization using the density method had a drawback: it creates gray areas, which had intermediate refractive index between those of materials used in a waveguide. This drawback results in optimized structures that are difficult to fabricate.

To suppress the gray areas, two types of approaches have been investigated; one is a density filtering technique [13], which has also been applied to optimization for optical devices such as photonic crystals [4]-[7], periodic grating surfaces [8], and plasmonic and dielectric structures [9], and the other is

T. Yasui, J. Sugisaka, and K. Hirayama are with the Department of Electrical and Electronic Engineering, Kitami Institute of Technology, Kitami 090-8507, Japan (e-mail: yasui@mail.kitami-it.ac.jp; sugisaka@ mail.kitamiit.ac.jp; hirakc@mail.kitami-it.ac.jp).

Y. Tsuji is with the Division of Information and Electronic Engineering, Muroran Institute of Technology, Muroran 050-8585, Japan (e-mail: ytsuji@mmm.muroran-it.ac.jp). a technique based on a projection scheme using Heaviside function [14]. The latter can be classified into two schemes; one is the geometry projection method [10] and the is the topology optimization method with function-expansion-based refractive index distribution [11], [12], and both have been applied to optimization for optical waveguide devices. However, these previous works were carried out in two dimensions. The limitations of optimization for optical circuits in two dimensions have not been unveiled.

In this paper, the topology optimization method with function-expansion-based refractive index distribution is extended to optimization for three-dimensional optical circuits. As numerical examples, the proposed method is applied to three-branch waveguides. We will see that an equally branching three-branch waveguide is successfully achieved using the proposed method, but that topology optimization in two dimensions fails to design three-dimensional three-branch waveguides.

In Section II, we review formulations of the proposed method. A few numerical results are shown in Section III. Section IV is the conclusion.

\section{TOPOLOGY OPTIMIZATION}

\section{A. Representation of Refractive Index Distribution in Design Region}

As an example, we consider a three-dimensional threebranch optical waveguide consisting of two materials with refractive indices of $n_{1}$ and $n_{2}$, as shown in Fig. 1. The computational region is surrounded by the perfectly matched layer (PML) [15]-[17]. A refractive index distribution in the design region of size $W_{x}$ by $W_{z}$ is topology optimized so that optical power input from port 1 equally branches into ports 2 to 4 .

In this paper, the refractive index distribution in the design region is expressed as follows [11]:

$$
\begin{gathered}
n^{2}(x, y, z)=n_{1}^{2}+\left(n_{2}^{2}-n_{1}^{2}\right) H(w(x, y, z)) \\
H(w)= \begin{cases}0 & (w \leq-h) \\
\frac{1}{2}\left(\frac{w+h}{h}\right)^{2} & (-h<w<0) \\
1-\frac{1}{2}\left(\frac{w-h}{h}\right)^{2} & (0 \leq w<h) \\
1 & (w \geq h)\end{cases}
\end{gathered}
$$




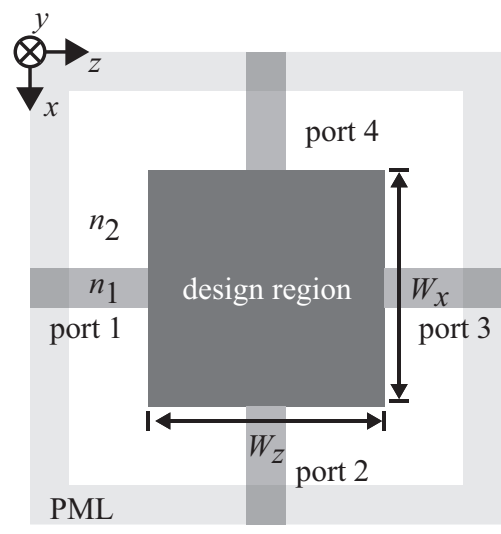

(a)

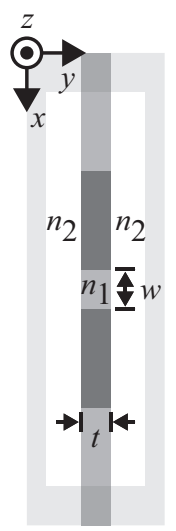

(b)
Fig. 1. Three-branch optical waveguide to be topology optimized: (a) top and (b) side views.

where $w(x, y, z)$ is some function to determine the refractive index distribution. The function $H(w)$ is used to convert a continuous value of $w(x, y, z)$ into either $n_{1}$ or $n_{2}$. Since $H(w)$ must be differentiable in the sensitivity analysis described bellow, $H(w)$ has a transition region of width $2 h$. The value of $h$ can be either fixed or variable through the optimization process. After the optimization process is converged, $h$ is reduced to zero to obtain an index distribution without gray area.

For $w(x, y, z)$, we employ the following sinusoidalfunction-based expression:

$$
\begin{aligned}
w(x, y, z) & =\sum_{i=0}^{N_{x}-1} \sum_{j=-N_{z}}^{N_{z}-1}\left(a_{i j} \cos \theta_{i j}+b_{i j} \sin \theta_{i j}\right) \\
\theta_{i j} & =\frac{2 \pi i}{L_{x}}\left(x-x_{0}\right)+\frac{2 \pi j}{L_{z}}\left(z-z_{0}\right)
\end{aligned}
$$

where $N_{x}$ and $2 N_{z}$ are the numbers of the expansion functions along the $x$ - and $z$-directions, respectively, $a_{i j}$ and $b_{i j}$, which are normalized as $\max (|w(x, y, z)|)=1$, are the expansion coefficients, and $x_{0}$ and $z_{0}$ are the $x$ - and $z$-coordinates of a given point in the computational region, respectively. Here, $L_{x}$ and $L_{y}$ are set to be greater than $W_{x}$ and $W_{y}$, respectively. To ensure the feasibility of optimized structures, $w(x, y, z)$ is assumed to be constant with respect to $y$ so that the refractiveindex-distribution in the design region will be invariant in depth.

\section{B. Finite Element Method}

Since evaluation of characteristics of a given optical device is necessary in topology optimization, the three-dimensional finite element method (FEM) using tetrahedral edge elements for a waveguide discontinuity problem [18] is employed to estimate the transmission characteristics. Applying the FEM, we obtain a final matrix equation as follows:

$$
[P]\{\Phi\}=\{\Psi\}_{\Gamma}
$$

where $[P]$ is a finite element matrix, $\{\Phi\}$ is an edge value vector of unknown electric/magnetic field, and $\{\Psi\}_{\Gamma}$ is a vector related to quantities on the incident plane. These matrix and vectors are described in detail in [18], [19].

For this example, a quarter of the solution domain is analyzed due to symmetry in the $x$ - and $y$-directions.

\section{Sensitivity Analysis}

In the topology optimization, we need to know the dependence of transmission characteristics on the expansion coefficients $a_{i j}$ and $b_{i j}$ in (3). The transmission characteristics are represented by scattering parameters $S_{n 1}(n=1, \ldots, N)$ given as follows:

$$
S_{n 1}=-\delta_{n 1}+\left\{g_{n}\right\}^{T}\{\Phi\}
$$

where $\delta_{n 1}$ represents the Kronecker delta, $\left\{g_{n}\right\}$ is a known vector related to a propagating mode in port $n, N$ is the number of ports, and superscript $T$ denotes a transpose.

To calculate the dependence of $S_{n 1}$ on $a_{i j}$ or $b_{i j}$, the adjoint variable method (AVM) [3]-[12], [20]-[25] is used. Let $C$ be an objective function to be minimized subject to the constraint (5). Here we define $C$ as

$$
C=F\left(\left|S_{11}\right|,\left|S_{21}\right|, \ldots,\left|S_{N 1}\right|\right)
$$

where $F$ stands for a function with respect to $\left|S_{11}\right|,\left|S_{21}\right|, \ldots$, $\left|S_{N 1}\right|$. We rewrite the objective function by adding the zero function:

$$
\begin{aligned}
\tilde{C}= & F\left(\left|S_{11}\right|,\left|S_{21}\right|, \ldots,\left|S_{N 1}\right|\right) \\
& -\operatorname{Re}\left[\{\gamma\}^{T}\left([P]\{\Phi\}-\{\Psi\}_{\Gamma}\right)\right]
\end{aligned}
$$

where $\{\gamma\}$ is any arbitrary complex vector. From (8), we obtain that

$$
\begin{aligned}
\frac{\partial \tilde{C}}{\partial c_{i j}}= & \operatorname{Re}\left[\left(\sum_{n=1}^{N} \frac{\partial F}{\partial\left|S_{n 1}\right|} \frac{S_{n 1}^{*}}{\left|S_{n 1}\right|}\left\{g_{n}\right\}^{T}-\{\gamma\}^{T}[P]\right) \frac{\partial\{\Phi\}}{\partial c_{i j}}\right. \\
& \left.-\{\gamma\}^{T} \frac{\partial[P]}{\partial c_{i j}}\{\Phi\}\right]
\end{aligned}
$$

where $c_{i j}$ is $a_{i j}$ or $b_{i j}$, and $*$ denotes complex conjugate. In turn, this can be written as

$$
\frac{\partial \tilde{C}}{\partial c_{i j}}=-\operatorname{Re}\left[\{\gamma\}^{T} \frac{\partial[P]}{\partial c_{i j}}\{\Phi\}\right]
$$

when $\{\gamma\}$ satisfies the adjoint equation:

$$
[P]^{T}\{\gamma\}=\sum_{n=1}^{N} \frac{\partial F}{\partial\left|S_{n 1}\right|} \frac{S_{n 1}^{*}}{\left|S_{n 1}\right|}\left\{g_{n}\right\} .
$$

After the sensitivity analysis, $a_{i j}$ and $b_{i j}$ are updated using the hill climbing method.

\section{NumericAl Results}

As numerical examples of the proposed design method, we consider the three-branch optical waveguide, as shown in Fig. 1. The refractive indices of core and cladding are $n_{1}=3.4$ and $n_{2}=1.45$, respectively. The waveguide width is $w=0.4 \mu \mathrm{m}$, the design-region width in $x$ - and $z$-directions are, respectively, $W_{x}=W_{z}=2 \mu \mathrm{m}$. Both the waveguide and design-region thicknesses are $t=0.2 \mu \mathrm{m}$. For (3) and (4), $N_{x}=N_{z}=16$, 
and $L_{x}=L_{z}=2.4 \mu \mathrm{m}$, where the dependence of $N_{x}(=$ $N_{z}$ ) on the optimization is demonstrated in Fig. 7 of [12]. Although the increasing numbers of $N_{x}$ and $N_{z}$ may improve the circuit performance, the degree of freedom in optimization also increases. This will cause fragment patterns in the design region, which decrease the feasibility of the circuits, and slow convergence on optimization. As we will see below in this section, the optimized circuits show acceptable performances with $N_{x}=N_{z}=16$. The operation wavelength is $\lambda=$ $1.55 \mu \mathrm{m}$. The fundamental $E^{x}$ mode is launched into port 1 . The objective function to be minimized is given as follows:

$$
C=1-\sum_{n=2}^{4}\left|S_{n 1}\right|^{2}+\left.\left|\frac{\left|S_{21}\right|^{2}+\left|S_{41}\right|^{2}}{2}-\right| S_{31}\right|^{2} \mid .
$$

We demonstrate optimum design of the three-branch waveguide in three ways. Each is described below.

\section{A. Two-dimensional optimization with fixed $h$ and its three- dimensional analysis}

First, we consider three-dimensional analysis for a threebranch waveguide obtained by the two-dimensional topology optimization [3]. To construct the three-dimensional model, the optimized structure in two dimensions is thickened to $t$. In this optimization, the value of $h$ in (2) is fixed to 0.005 . We will see that topology optimization in three dimensions is necessary.

The results for two-dimensional topology optimization are shown in Figs. 2 and 3. The effective index method is applied to obtain the two-dimensional model, thus refractive indices of the core and cladding are 2.70332 and 1.45 , respectively. Fig. 2 shows the initial and optimized structures together with propagating field distributions. The normalized power as a function of iteration count is shown in Fig. 3. The optimum structure is obtained at the 193rd iteration. The normalized output powers are $\left|S_{21}\right|^{2}=0.322,\left|S_{31}\right|^{2}=0.332$, and $\left|S_{41}\right|^{2}=0.322$. We can see that optical power is almost equally branched, and that the transmission characteristics are drastically improved through the optimization. Note that, in this section, the resulting normalized output power is the one for the optimized structures before the elimination of gray area. We will see in Subsection III-C that the effect caused by the elimination of gray area can be negligible.

However, the propagating field distribution in the threedimensional model built from Fig. 2 (b) is shown in Fig. 4. The normalized output power are $\left|S_{21}\right|^{2}=\left|S_{41}\right|^{2}=0.019$ and $\left|S_{31}\right|^{2}=0.441$. In the three-dimensional analysis, the computational region was reduced to quarter because of the symmetry, as a result $\left|S_{21}\right|^{2}=\left|S_{41}\right|^{2}$. The transmission characteristics of the three-dimensional model is obviously deteriorated. Thus topology optimization for three-dimensional optical circuits is necessary.

\section{B. Optimization for three-dimensional optical circuits with fixed $h$}

In this subsection, the three-dimensional three-branch waveguide is optimized. The two-dimensionally optimized

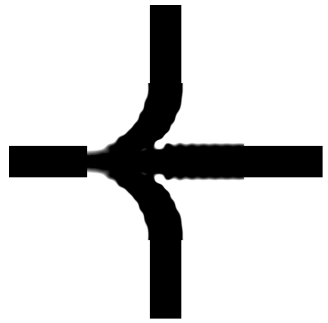

(a)

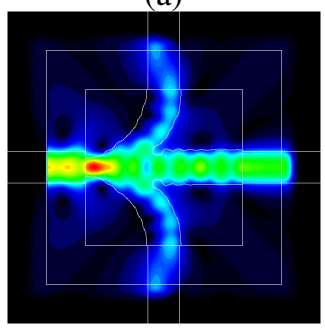

(c)

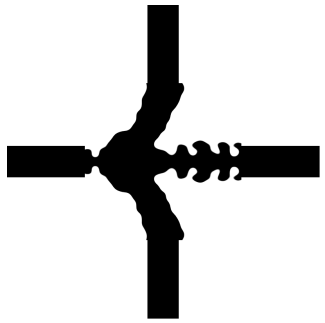

(b)

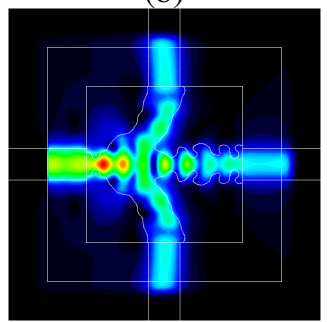

(d)
Fig. 2. Two-dimensional topology optimization for the three-branch waveguide: (a) the initial, and (b) the optimized structures, propagating field in (c) the initial and (d) the optimized structures.

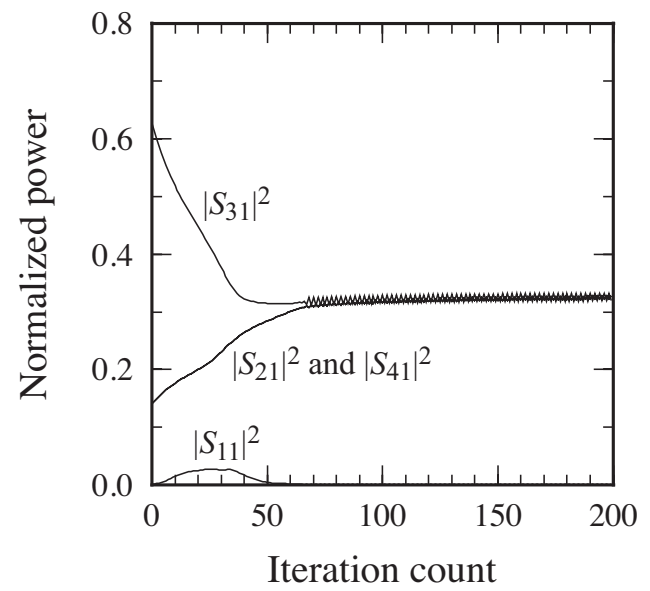

Fig. 3. Normalized power as a function of iteration count for topology optimization in two dimensions. The optimum structure is obtained at the 193rd iteration. It is noted that the curves for $\left|S_{21}\right|^{2}$ and $\left|S_{41}\right|^{2}$ are overlapped.

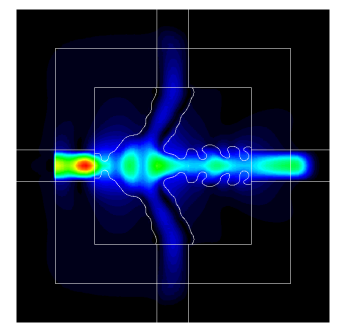

Fig. 4. Propagating field in the three-dimensional three-branch waveguide, where the optimum structure obtained by the two-dimensional optimization is thickened to $t=0.2 \mu \mathrm{m}$.

waveguide shown in Fig. 2 (b) is taken as the initial structure to reduce the computational time. The computational region is reduced because of the symmetry. Here, the value of $h$ is fixed to 0.005 . 


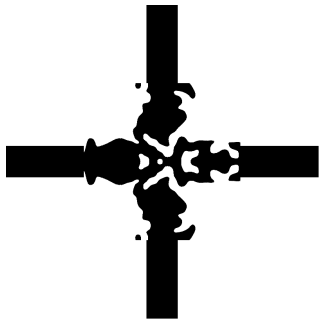

(a)

(c)

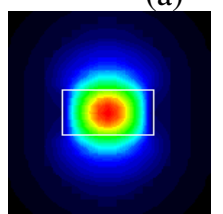

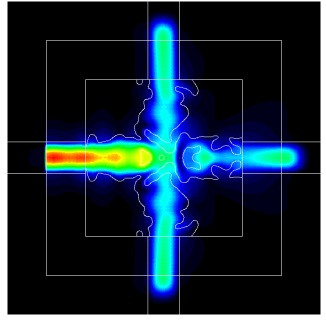

(b)

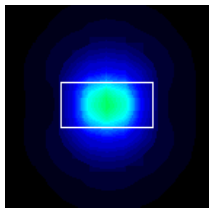

(d)

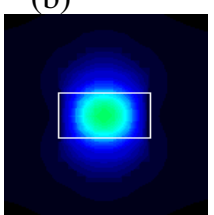

(e)
Fig. 5. Numerical results for the topology optimization with a fixed value of $h$. (a) The optimized structure. (b) Top view of propagating field distribution. Cross-sectional views of propagating field distributions at (c) port 1, (d) port 2 and 4 [because of the symmetry], and (e) port 3.

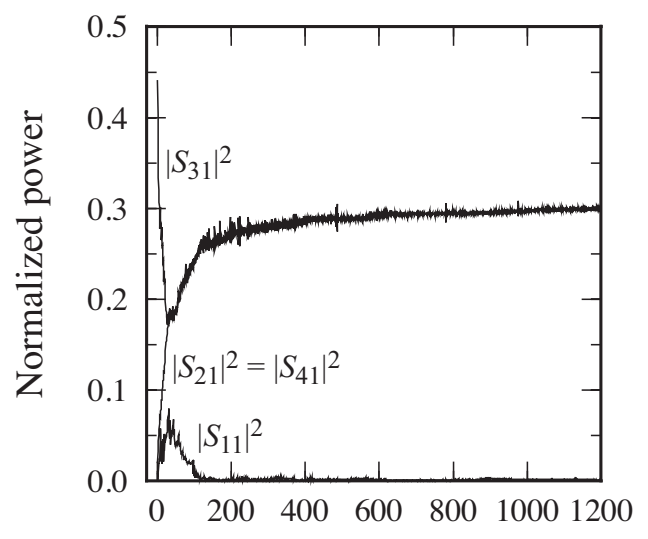

Iteration count

Fig. 6. Normalized power as a function of iteration count for topology optimization with a fixed value of $h$. The optimum structure is obtained at the 1198th iteration. We note that $\left|S_{21}\right|^{2}=\left|S_{41}\right|^{2}$ because of the symmetry.

The optimized structure and propagating field distributions in it are shown in Fig. 5, and normalized power as a function of iteration count is shown in Fig. 6. The optimum structure was obtained at the 1198th iteration, and its normalized output powers are $\left|S_{21}\right|^{2}=\left|S_{41}\right|^{2}=0.322$ and $\left|S_{31}\right|^{2}=0.332$. We can see that the transmission characteristics are much improved by using the three-dimensional topology optimization.

\section{Optimization for three-dimensional optical circuits vari- able $h$}

Next, we consider topology optimization of the threedimensional three-branch waveguide in the same way as the previous subsection, but the value of $h$ is renewed as a monotonically decreasing function of iteration count $n$ like a decreasing radius of density filter [9], as follows:

$$
h=2 \exp (-n / 200) \text {. }
$$

The optimized structure and propagating field distributions in it are shown in Fig. 7, and normalized power as a function

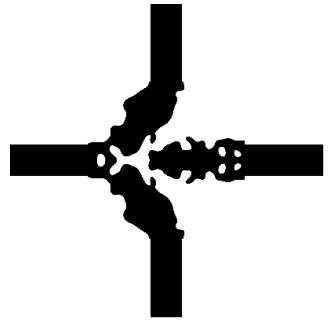

(a)

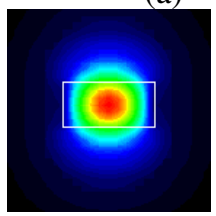

(c)

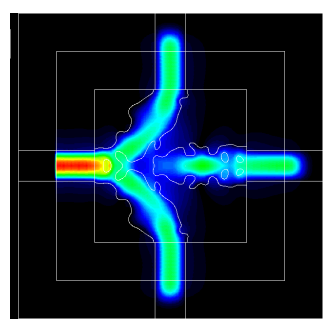

(b)

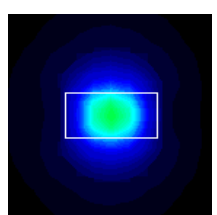

(d)

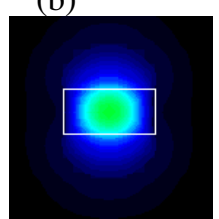

(e)
Fig. 7. Numerical results for the topology optimization with variable value of $h$. (a) The optimum structure. (b) Top view of propagating field distribution. Cross-sectional views of propagating field distributions at (c) port 1, (d) port 2 and 4 [because of the symmetry], and (e) port 3.

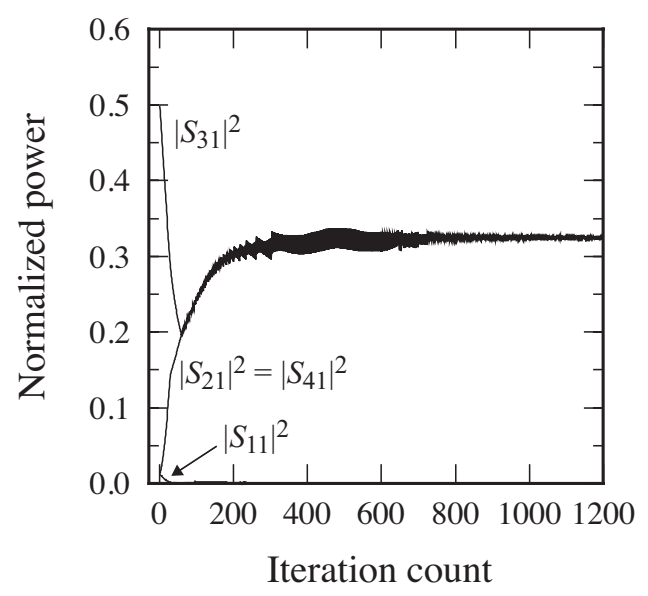

Fig. 8. Normalized power as a function of iteration count for topology optimization with variable value of $h$ given as (13). The optimum structure is obtained at the 1161 st iteration. We note that $\left|S_{21}\right|^{2}=\left|S_{41}\right|^{2}$ because of the symmetry.

of iteration count is shown in Fig. 8. The optimum structure was obtained at the 1161st iteration, and its normalized output powers are $\left|S_{21}\right|^{2}=\left|S_{31}\right|^{2}=\left|S_{41}\right|^{2}=0.325$. An equally branching three-blanch waveguide is obtained. We think that the better solution than the one in the previous subsection has been obtained because of higher degree of freedom for modification of the structure in early step of the optimization due to larger gray area.

The optimized structures before the elimination of gray area for Fig. 7 (a) is shown in Fig. 9. The gray area, which occupies only $0.63 \%$ of area of the design region, is painted in red. The normalized output power shown above is for a structure with gray area. After the elimination of gray area [Fig. 7 (a)], the normalized output powers become $\left|S_{21}\right|^{2}=\left|S_{41}\right|^{2}=0.3248$ and $\left|S_{31}\right|^{2}=0.3251$. The difference by the elimination of gray area can be negligible. 


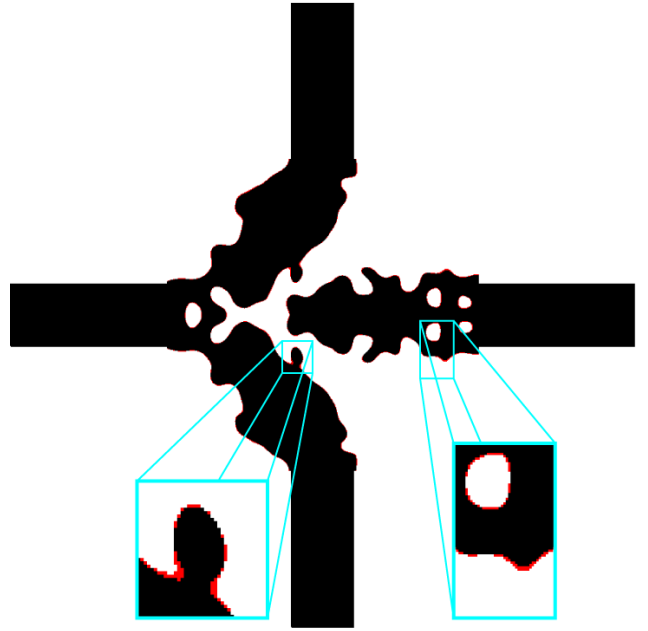

Fig. 9. The optimized structure before the elimination of gray area for Fig. 7 (a). The gray area, which occupies $0.63 \%$ of area of the design region, is painted in red. Insets are magnified refractive index distributions.

\section{Initial-structure dependency}

Finally, we consider initial-structure dependency on the topology optimization.

The three-branch optical waveguide is optimized with a simpler initial structure as shown in Fig. 10 (a), where the design region has a constant refractive index of 2.921. Here, optimization with a fixed value of $h$ of 0.005 for a threedimensional optical circuit is carried out from the start. The optimum structure is obtained at the 196th iteration as shown in Fig. 10 (b), and propagating field in it is shown in Fig. 10 (c). Here, its normalized output powers are $\left|S_{21}\right|^{2}=\left|S_{41}\right|^{2}=$ 0.241 and $\left|S_{31}\right|^{2}=0.338$.

In comparison with the previous results, we can see that the optimized results strongly depend on initial structures. We think that there are a lot of local optima due to a highindex-contrast waveguide structure, and that the result was trapped in one of them. Thus an initial structure with a starting guess is needed for successful convergence to optimize highindex-contrast waveguide structures for the suggested topology optimization approach.

\section{CONCLUSION}

We extended the topology optimization method with function-expansion-based refractive index distribution to optimization for three-dimensional optical circuits. The proposed method was applied to design equally branching three-branch waveguides, and it has been achieved. We have also found out that the optimization for a three-dimensional optical circuit is necessary for optimum design of three-dimensional waveguides.

The authors would like to thank Mr. Ryosuke Kijima for his valuable work.

\section{REFERENCES}

[1] J.S. Jensen and O. Sigmund, "Systematic design of photonic crystal structures using topology optimization: Low-loss waveguide bends," Appl. Phys. Lett., vol. 84, no. 12, pp. 2022-2024, Mar. 2004.

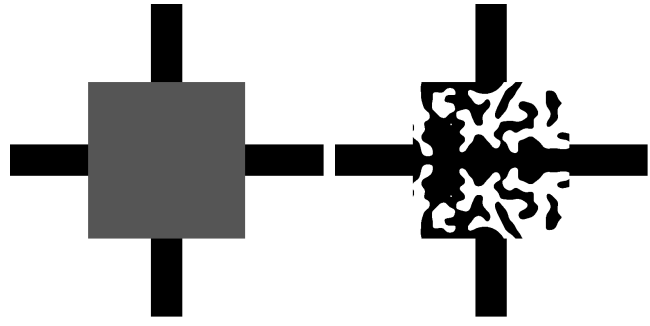

(a)

(b)

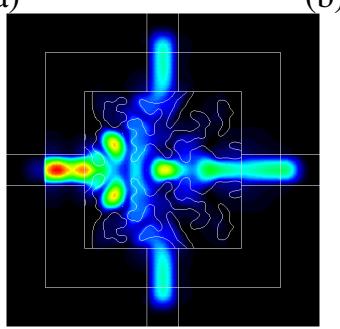

(c)

Fig. 10. Numerical results for the topology optimization for the threedimensional optical circuit with a simple initial structure: (a) the initial and (b) optimized structures, and (c) propagating field in the optimized structure.

[2] J. Jensen, O. Sigmund, L.H. Frandsen, P.I. Borel, A. Harpoth, and M. Kristensen, "Topology design and fabrication of an efficient double $90^{\circ}$; photonic crystal waveguide bend," IEEE Photon. Technol. Lett., vol. 17, no. 6, pp. 1202-1204, Jun. 2005.

[3] Y. Tsuji, K. Hirayama, T. Nomura, K. Sato, and S. Nishiwaki, "Design of optical circuit devices based on topology optimization," IEEE Photon. Technol. Lett., vol. 18, no. 7, pp. 850-852, Apr. 2006.

[4] R. Matzen, J.S. Jensen, and O. Sigmund, "Topology optimization for transient response of photonic crystal structures," J. Opt. Soc. Am. B, vol. 27, pp. 2040-2050, Oct. 2010.

[5] F. Wang, J.S. Jensen, and O. Sigmund, "Robust topology optimization of photonic crystal waveguides with tailored dispersion properties," $J$. Opt. Soc. Am. B, vol. 28, pp. 387-397, Mar. 2011.

[6] R. Matzen, J.S. Jensen, and O. Sigmund, "Systematic design of slowlight photonic waveguides," J. Opt. Soc. Am. B, vol. 28, pp. 2374-2382, Oct. 2011.

[7] F. Wang, J.S. Jensen, J. Mørk, and O. Sigmund, "Systematic design of loss-engineered slow-light waveguides," J. Opt. Soc. Am. A, vol. 29, pp. 2657-2666, Dec. 2012.

[8] K.S. Friis and O. Sigmund, "Robust topology design of periodic grating surfaces," J. Opt. Soc. Am. B, vol. 29, pp. 2935-2943, Oct. 2012.

[9] M.B. Dühring and O. Sigmund, "Optimization of extraordinary optical absorption in plasmonic and dielectric structures," J. Opt. Soc. Am. B, vol. 30, pp. 1154-1160, May 2013.

[10] W.R. Frei, D.A. Tortorelli, and H.T. Johnson, "Geometry projection method for optimizing photonic nanostructures," Opt. Lett., vol. 32, pp. 77-79, Jan. 2007.

[11] Y. Tsuji and K. Hirayama, "Design of optical circuit devices using topology optimization method with function-expansion-based refractive index distribution," IEEE Photon. Technol. Lett., vol. 20, no. 12, pp. 982-984, Jun. 2008.

[12] K. Fujimoto, Y. Tsuji, K. Hirayama, T. Yasui, S. Sato, and R. Kijima, "A study on topology optimization of optical circuits consisting of multimaterials," J. Lightw. Technol., vol. 30, no. 13, pp. 2210-2215, Jul. 2012.

[13] O. Sigmund, "Morphology-based black and white filters for topology optimization," Struct. Multidisc. Optim., vol. 33, pp. 401-424, 2007.

[14] J.K. Guest, J.H. Prévost, and T. Belytschko, "Achieving minimum length scale in topology optimization using nodal design variables and projection functions," Int. J. Numer. Meth. Engng., vol. 61, pp. 238-254, 2004.

[15] J.-P. Berenger, "A perfectly matched layer for the absorption of electromagnetic waves," J. Comput. Phys., vol. 114, no. 2, pp. 185-200, Oct. 1994.

[16] F. Teixeira and W. Chew, "General closed-form PML constitutive tensors to match arbitrary bianisotropic and dispersive linear media," IEEE Microw. Guided Wave Lett., vol. 8, no. 6, pp. 223-225, Jul. 1998. 
[17] N. Kono and Y. Tsuji, "Oriented perfectly matched layer with flexible parameters for waveguide discontinuity problems," IEEE Photon. Technol. Lett., vol. 16, no. 4, pp. 1089-1091, Apr. 2004.

[18] Y. Ishizaka, Y. Kawaguchi, K. Saitoh, and M. Koshiba, "Threedimensional finite-element solutions for crossing slot-waveguides with finite core-height," J. Lightw. Technol., vol. 30, no. 21, pp. 3394-3400, Nov. 2012.

[19] Y. Tsuji and M. Koshiba, "Finite element method using port truncation by perfectly matched layer boundary conditions for optical waveguide discontinuity problems," J. Lightw. Technol., vol. 20, no. 3, pp. 463-468, Mar. 2002.

[20] F. Alessandri, M. Mongiardo, and R. Sorrentino, "New efficient full wave optimization of microwave circuits by the adjoint network method," IEEE Microw. Guided Wave Lett., vol. 3, no. 11, pp. 414416, Nov. 1993.

[21] I.-H. Park, I.-G. Kwak, H.-B. Lee, S. yop Hahn, and K.-S. Lee, "Design sensitivity analysis for transient eddy current problems using finite element discretization and adjoint variable method," IEEE Trans. Magn., vol. 32, no. 3, pp. 1242-1245, May 1996.

[22] H.-B. Lee and T. Itoh, "A systematic optimum design of waveguide-tomicrostrip transition," IEEE Trans. Microw. Theory Tech., vol. 45, no. 5 , pp. 803-809, May 1997.

[23] H. Akel and J. Webb, "Design sensitivities for scattering-matrix calculation with tetrahedral edge elements," IEEE Trans. Magn., vol. 36, no. 4, pp. 1043-1046, Jul. 2000.

[24] N. Nikolova, J. Zhu, D. Li, M. Bakr, and J. Bandler, "Sensitivity analysis of network parameters with electromagnetic frequency-domain simulators," IEEE Trans. Microw. Theory Tech., vol. 54, no. 2, pp. 670681, Feb. 2006

[25] K. Hirayama, Y. Tsuji, S. Yamasaki, and S. Nishiwaki, "Design optimization of H-plane waveguide component by level set method," IEICE Trans. Electron., vol. E94-C, no. 5, pp. 874-881, May 2011.

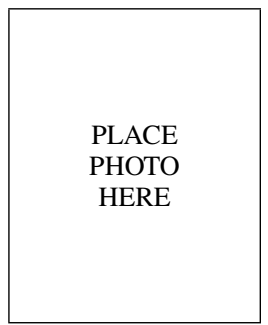

Yasuhide Tsuji (M' 97) received the B.S., M.S., and $\mathrm{Ph} . \mathrm{D}$. degrees in electronic engineering from Hokkaido University, Sapporo, Japan, in 1991, 1993, and 1996, respectively.

In 1996, he joined the Department of Applied Electronic Engineering, Hokkaido Institute of Technology, Sapporo, Japan. From 1997 to 2004, he was an Associate Professor of Electronics and Information Engineering at Hokkaido University. From 2004 to 2011, he was an Associate Professor of Electrical and Electronic Engineering at Kitami Institute of Technology, Kitami, Japan. Since 2011, he has been a Professor of Information and Electronic Engineering at Muroran Institute of Technology, Muroran, Japan. He has been interested in wave electronics.

Dr. Tsuji is a member of the Institute of Electronics, Information and Communication Engineers (IEICE), the Japan Society of Applied Physics, the Optical Society of America (OSA), and IEEE. In 1997 and 1999, he was awarded the Excellent Paper Award from IEICE. In 2000, he has received the Third Millennium Medal from IEEE.

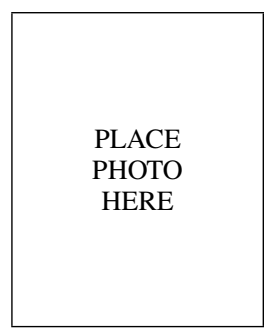

Jun-ichiro Sugisaka received the BE, ME, and $\mathrm{Ph} . \mathrm{D}$. degree in nanophotonics from University of Tsukuba, Tsukuba, Japan, in 2005, 2007, and 2010, respectively. From 2008 to 2010, he was a Research Fellow of the Japan Society for the Promotion Science. From 2010 to 2013, he joined Center for Optical Research Education in Utsunomiya University, Utsunomiya, Japan as a Doctoral Research Fellow. Since then he has been an Assistant Professor of the Department of Electrical and Electronic Engineering, Kitami Institute of Technology, Kitami, Japan.

His research interests are in photonic crystal, diffraction, scattering theory and their computer simulation, and works for developing integrated lightwave circuits and novel simulation algorithms.

Dr. Sugisaka is a member of the Japan Society of Applied Physics, Optical Society of Japan, the Institute of Electronics, Information, and Communication Engineers (IEICE) of Japan.

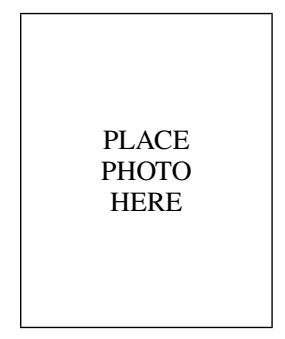

Takashi Yasui ( $S^{\prime}$ 00-M' 02) received the B.S degree in electronic engineering from Fukui University, Fukui, Japan, in 1997, and the M.S. and Ph.D. degrees in electronic engineering from Hokkaido University, Sapporo, Japan, in 1999 and 2001, respectively.

From 1999 to 2002, he was a Research Fellow of the Japan Society for the Promotion of Science. In 2002, he joined Fujitsu Ltd., Chiba, Japan. From 2004 to 2011, he was an Assistant Professor of the Department of Electronic and Control Systems Engineering, Shimane University, Matsue, Japan. Since 2011, he has been an Associate Professor of the Department of Electrical and Electronic Engineering, Kitami Institute of Technology, Kitami, Japan. He has been engaged in research on wave electronics.

Dr. Yasui is a member of the Applied Computational Electromagnetics Society, the Optical Society of America, the Institute of Electronics, Information, and Communication Engineers (IEICE) of Japan, and the Magnetics Society of Japan.

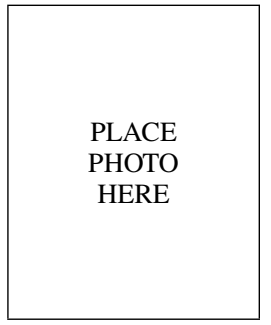

Koichi Hirayama (SM' 96) received the B.S., M.S., and $\mathrm{Ph} . \mathrm{D}$. degrees in electronic engineering from Hokkaido University, Sapporo, Japan, in 1984, 1986, and 1989 , respectively.

In 1989, he joined the Department of Electronic Engineering, Kushiro National College of Technology, Kushiro, Japan. In 1992, he became an Associate Professor of Electronic Engineering at Kitami Institute of Technology, Kitami, Japan, and in 2004 he became a Professor. He has been interested in the optical waveguides. analysis and optimal design of electromagnetic and

Dr. Hirayama is a member of the Institute of Electronics, Information and Communication Engineers (IEICE) of Japan and the Japan Society of Applied Physics. 